\title{
Kyphomelic dysplasia
}

INSERM

\section{Source}

INSERM. (1999). Orphanet: an online rare disease and orphan drug data base.

Kyphomelic dysplasia. ORPHA:1801

A rare primary bone dysplasia characterized, radiologically, by short, stubby long bones, severely angulated femurs and lesser bowing of other long bones (mild, moderate or no bowing), short and wide illiac wings with horizontal acetabular roofs, platyspondyly and a narrow thorax, clinically manifesting with severe, disproportionate short stature.

Regression of femora angulation is observed with advancing age. 